\title{
Effect of Flow on Various Helmholtz Resonators
}

\author{
Hyunsu Kim \\ Department of Automotive Engineering, Dong-Eui University, 176 Eomgwang-ro, Busan, South Korea, 47340.
}

\begin{abstract}
Iljae Lee
Department of Mechanical Engineering, Jeonbuk National University, 567 Baekje-daero, Jeonju-si, Jeollabuk-do, South Korea.
\end{abstract}

(Received 16 August 2018; accepted 9 January 2019)

The acoustic performance of Helmholtz resonators with different configurations in the presence of mean flow is experimentally investigated. The transmission loss of Helmholtz resonators is measured using a flow-impedance tube setup to identify mean flow effects. First, Helmholtz resonators under two different flow conditions, grazing and direct flow, are considered at $\mathrm{Ma}=0.05$ and 0.1 . Then the impact of mean flow on the transmission loss of Helmholtz resonators with leakage holes is also demonstrated. For Helmholtz resonators with leakage holes, single peak of transmission loss is identified at $\mathrm{Ma}=0.0$ and 0.1 . However, two peaks appear at $\mathrm{Ma}=0.05$ as the number of opening holes increases. The experimental results show that the effect of mean flow strongly depends on the configurations of Helmholtz resonators and the conditions of mean flow. Thus the variation of acoustic performance due to mean flow should be considered in the design of Helmholtz resonators.

\section{NOMENCLATURE}

$\begin{array}{ll}H R & \text { Helmholtz resonator } \\ A_{u} & \text { sound pressure of incident wave } \\ A_{d} & \text { sound pressure of transmitted wave } \\ S_{11} & \text { auto-power spectrum of microphone } 1 \\ S_{33} & \text { auto-power spectrum of microphone } 3 \\ R_{d} & \text { reflection coefficients at the downstream side } \\ R_{u} & \text { reflection coefficients at the upstream side } \\ c_{0} & \text { speed of sound } \\ A & \text { cross-sectional area of the neck } \\ L_{e f f} & \text { effective neck length accounting for } \\ & \text { the end correction effect } \\ V & \text { cavity volume } \\ p & \text { acoustic pressure } \\ u & \text { acoustic velocity } \\ R & \text { acoustic resistance } \\ X & \text { acoustic reactance } \\ \rho_{0} & \text { density of air } \\ S_{d} & \text { cross-sectional area of a neck. }\end{array}$

\section{INTRODUCTION}

The Helmholtz resonator (HR) is one of the most popular silencers utilized in the intake and exhaust systems of vehicles to reduce noise at low frequency with relatively small volume. The acoustic performance of HR can be effectively adjusted with different neck and cavity geometries. Thus, variation of HRs are widely investigated by numerous researchers ${ }^{1-7}$ after Rayleigh's original work on HR. ${ }^{8}$ These studies focused mainly on the effect of variations of neck and volume on the acoustics performance in the absence of mean flow.

However, as the silencers are used in the presence of mean flow in the majority of applications, the effect of flow can be critical because the mean flow can alter the acoustic perfor- mance of the silencers. After Sivian ${ }^{9}$ investigated the effect of flow on a small orifice, numerous studies on HRs in the presence of flow were published. ${ }^{10-16}$ These studies, however, focused on insertion loss or acoustic impedance without demonstrating the transmission loss (TL).

In automotive intake and exhaust systems, a neck of HR may be integrated into the baffle due to the restraint in space. For these reasons, HR can be exposed to either direct or grazing flow. Kim and Selamet developed a flow-impedance tube setup that measured TL with flow. They investigated a single HR with grazing flow and found a dramatic impact on TL. ${ }^{17}$ Sun et al. compared the acoustic impedance for orifices with grazinginflow and grazing-outflow. ${ }^{18}$ They reported that the resistance had less magnitude with grazing-outflow than grazing-inflow. Selamet et al. investigated a HR with direct flow using Computational Fluid Analysis (CFD). However, they did not compare the effect of different flow directions casued by different neck configurations. ${ }^{19,20}$ TL prediction with flow using a commercial prediction tool is reported. The number of applications reported are limited. ${ }^{21}$

It is generally assumed that HR is sealed so that a cavity can communicate with main duct through only a neck. However, in practice, leakage holes may exist due to drain holes. In this case, additional paths between the cavity and main duct exist. These additional paths may change the acoustics behavior of HR. Selamet et al. investigated practical design of HR which has leakage holes. ${ }^{22}$ The leakage holes can be found in an exhaust muffler for draining water. They demonstrated that resonances of HR are dramatically changed by the holes. However, the study is limited to no-flow condition. Therefore, this paper investigates the effect of flow on key elements, such as HR with leakage and HR with different flow paths, using the flow-impedance tube setup that was introduced by Kim and Selamet. ${ }^{17}$ 


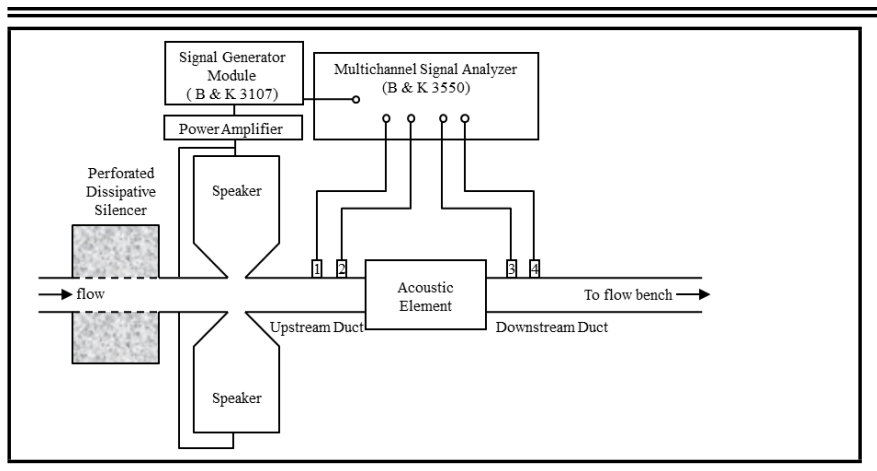

Figure 1. Flow-impedance tube setup (Kim and Selamet, 2011) ${ }^{17}$

Following this introduction, Section 2 illustrates the experimental setup and the HR configurations with different flow paths and with leakage. Section 3 discusses the results with TL, followed by conclusion with final remarks in Section 4.

\section{EXPERIMENTAL SETUP HELMHOLTZ RESONATORS}

AND

\section{EXPERIMENTAL SETUP}

Figure 1 illustrates the flow-impedance tube setup. The flow-impedance tube was developed to measure TL in the presence of flow by Kim and Selamet. ${ }^{17}$ While the details of the experimental setup have been described by Kim and Selamet ${ }^{17}$ extensively, a brief explanation is introduced here for readers' convenience. Two speakers of $100 \mathrm{~W}$ were used for sound input into the system. Various types of signals were generated by a B\&K 3107 signal generator, amplified, and then fed into the speakers. Four $1 / 4$ inch condenser microphones were flush-mounted into the duct to measure the acoustic pressure at upstream and downstream ducts with $4 \mathrm{~cm}$ microphone spacing, which ensured accuracy up to $3.4 \mathrm{kHz}$. The diameter of the main duct was $4.9 \mathrm{~cm}$, which provided a valid plane wave assumption of up to approximately $4 \mathrm{kHz}$. An acoustic element to be tested was placed in the middle of the duct. A B\&K 3550 multichannel signal analyzer was used to calculate the TL of the acoustic element by collecting and processing the sound pressure measured by the microphones. The downstream end was connected to a flow bench, which produced a pressure drop of up to $8.72 \mathrm{kPa}$. At the end of the upstream duct, a perforated dissipative silencer is installed to reduce the flow noise induced at the entrance of flow path.

To calculate the TL, the incident wave to the acoustic element in the upstream ducts and the transmitted wave from the acoustic element to downstream duct was obtained. The wave decomposition method was applied to measure the incident wave at the upstream pipe. ${ }^{23-25}$ To extract the transmitted wave at the downstream duct, a $30 \mathrm{~m}$ long flexible tube was adapted.

A transient signal, such as a burst random or a burst sine with a time duration of 0.1 second, was selected and then only a transmitted wave was obtained before the reflected wave at the end of a long flexible tube arrived at the downstream microphones. Following this, 100 time ensembles were used to improve the signal to noise ratio. Additionally, to avoid the mi- crophone mismatch in phase, calibration suggested by Chung and Blaser is applied. ${ }^{23,24}$ Then, the TL can be obtained as:

$$
T L=10 \log _{10}\left(\frac{A_{u}}{A_{d}}\right)^{2}=10 \log _{10}\left(\frac{S_{11}\left(1+R_{d}\right)^{2}}{S_{33}\left(1+R_{u}\right)^{2}}\right) ;
$$

where $A_{u}$ and $A_{d}$ represented the sound pressure of both the incident and transmitted waves. $S_{11}$ and $S_{33}$ represented the auto-power spectrum of microphones 1 and 3. $R_{d}$ and $R_{u}$ represented the reflection coefficients at the downstream and upstream sides.

\subsection{Configurations Resonators}

\section{of Helmholtz}

\subsubsection{Helmholtz resonators with direct and grazing flow}

Figure 2 shows the schematics of HRs with different orientations that were relative to flow direction. The dimensions of the neck and cavity were identical. In general, the resonance frequency of HR was determined by the dimensions of the neck and cavity with a well known equation as:

$$
f=\frac{c_{0}}{2 \pi} \sqrt{\frac{A}{L_{e f f} V}}
$$

where $c_{0}$ represented the speed of sound, $A$ represented the cross-sectional area of the neck, $L_{e f f}$ represented the effective neck length incorporating the end correction effect, and $V$ represented the cavity volume. Since the dimensions of the neck and cavity of both HRs were the same, the peak TL was expected to be almost identical in magnitude and frequency in the absence of mean flow. However, two HRs were subject to different flow paths, as shown in Figs. 2 (a) and (b).

\subsubsection{Helmholtz resonator with leakage}

Figure 3 illustrates an HR model with leakage studied by Selamet et al. ${ }^{22}$ Main chamber and HR volume were divided by a baffle and a neck was attached on the baffle. Up to 13 leakage holes, with $0.5 \mathrm{~cm}$ in diameter on the baffle as shown in Fig. 3 (b), could either be open or closed. Selamet et al. investigated the effect of the leakage holes on the TL in the absence of mean flow. ${ }^{22}$ Selamet et al. demonstrated that the resonance shifts to a higher frequency and the magnitude of the TL at resonance decreases with the hole opening and then increases as more holes open. When flow is considered, the majority of flow would pass through the main duct. Due to the fact that the HR cavity had two paths, which were neck and leakage holes, the flow path was more complicated, as indicated by arrows in Fig. 3 (a).

\section{RESULTS AND DISCUSSION}

\subsection{HR with Direct and Grazing Flow}

Figure 4 compares the measured TL of HRs with different flow conditions, grazing and direct flow, as illustrated in Fig. 2. Three different flow rates, $\mathrm{Ma}=0.0,0.05$ and 0.1 are 


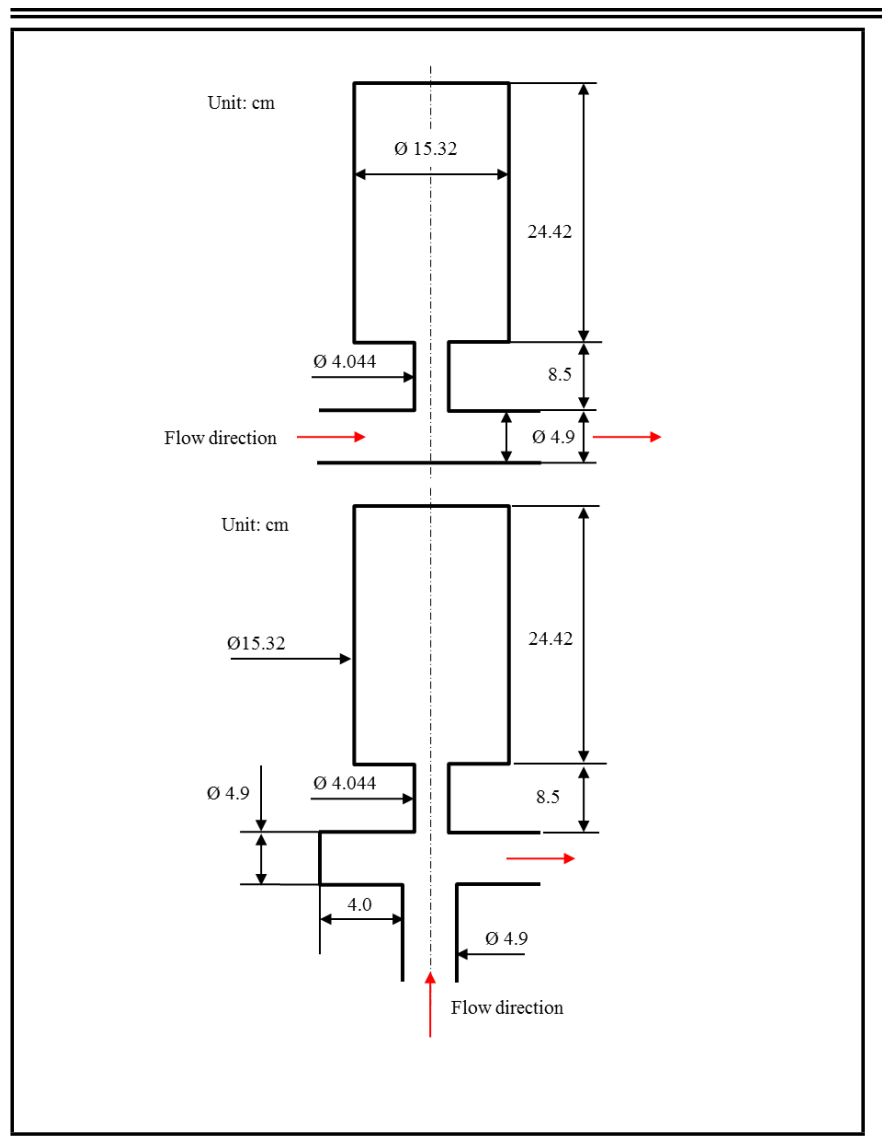

Figure 2. Schematics of HRs with different configuration in flow direction: (a) grazing flow; (b) direct flow.

applied for the measurement. Since the diameter of the main duct is $4.9 \mathrm{~cm}$, the volume velocities are calculated as 0.032 and $0.064 \mathrm{~m}^{3} / \mathrm{s}$ for $\mathrm{Ma}=0.05$ and 0.1 , respectively. Due to the limitation of the experimental set up, the maximum flow rate is up to $\mathrm{Ma}=0.1$. Even though the flow rate of $\mathrm{Ma}=0.1$ may be not enough to cover the flow rate in all practical applications, it is a meaningful flow rate for small size engines and/or at low engine rpm. In the absence of mean flow $(\mathrm{Ma}=0.0)$, as expected from the same dimensions in both the neck and cavity, TLs for two different configurations of HR are almost identical with peak frequency at $86 \mathrm{~Hz}$. Figure 4 also shows that, in the absence of mean flow, the TL predicted by one dimensional analysis agrees with the experiments. ${ }^{17}$ However, in the presence of mean flow, simple acoustic theory may not be applied to predict the TL and, thus experimental investigations have been utilized. As illustrated earlier by Kim and Selamet, ${ }^{17}$ the TL of HR with grazing flow shows dramatic changes with an increase in peak frequency and a decrease in peak magnitude as flow rate increases. They experimentally demonstrated the changes in the acoustic impedance, which can be expressed as:

$$
Z=\frac{p}{u}=R+i X
$$

where $p$ is the acoustic pressure, $u$ the velocity, $R$ the resistance, and $X$ the reactance. They showed that, for HR with grazing flow shown in Fig. 2 (a), the acoustic resistance increases and the reactance decreases as flow rate increases. These changes in acoustic impedance caused by grazing flow lead to an increase in peak TL frequency and a decrease in peak TL magnitude. The relationship between TL and the acoustic

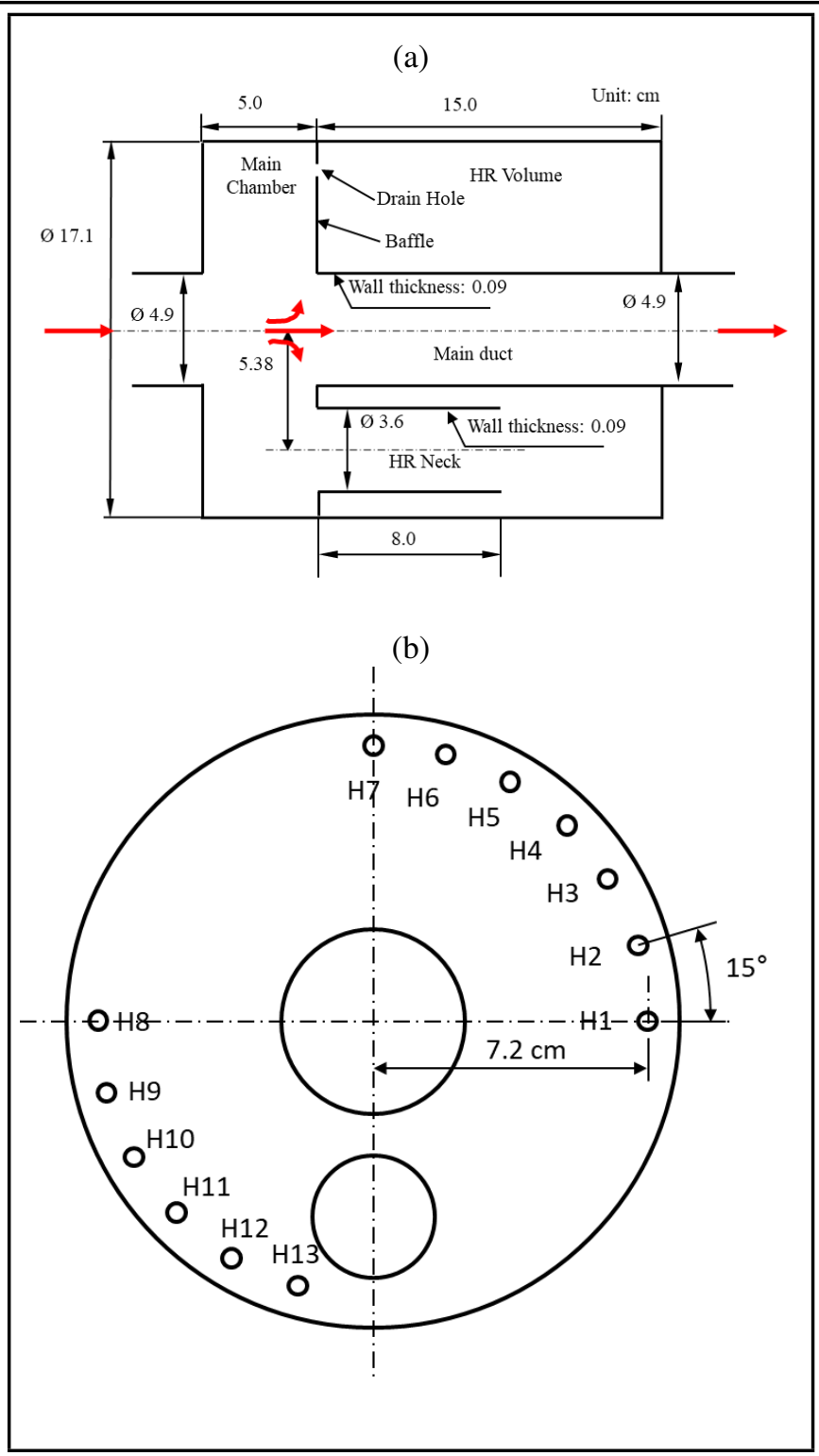

Figure 3. The schematic of HR with leakage: (a) dimensions; (b) baffle with leakage holes (Selamet et al., 2011). ${ }^{22}$

impedance can be expressed as:

$$
T L=20 \log _{10}\left|\frac{\rho_{0} c_{0}}{2 S_{d} Z}+1\right| ;
$$

where $\rho_{0}$ is the density of air and $S_{d}$ the cross-sectional area of a neck.

Figure 4 shows that, compared to the behavior of HR under grazing flow, the HR with direct flow presents significantly different characteristics, especially at $\mathrm{Ma}=0.05$. The magnitude of the TL sustains the level and the peak shifts to lower frequency with $\mathrm{Ma}=0.05$. For example, studies on the acoustic behavior of flow through an orifice were done by Baumeister and Rice, ${ }^{14}$ Hersch and Walker, ${ }^{15}$ and Sun et al. ${ }^{18}$ In these previous studies, the flow goes through an orifice, which is noted as either 'inflow' or 'outflow' by Sun et al. ${ }^{18}$ However, 'direct flow' in Fig. 4 is different from the flow conditions of the previous studies. The 'direct flow' in this study does not go through the neck. For $\mathrm{Ma}=0.1$ with direct flow, the magnitude of the TL decreases in a manner that is comparable to the HR with grazing flow. However, unlike the grazing flow, the peak 


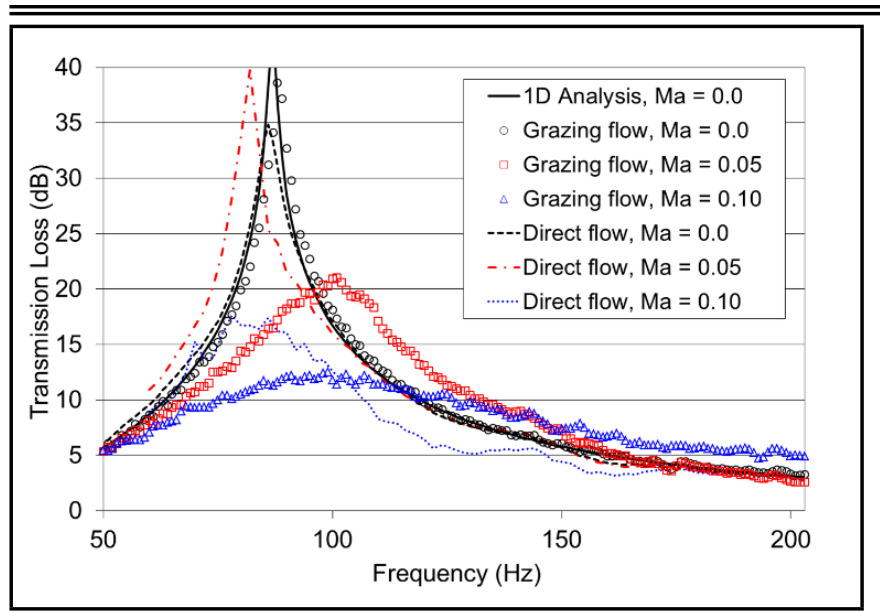

Figure 4. Comparison of TL in the presence of flow between HR with grazing flow and direct flow.

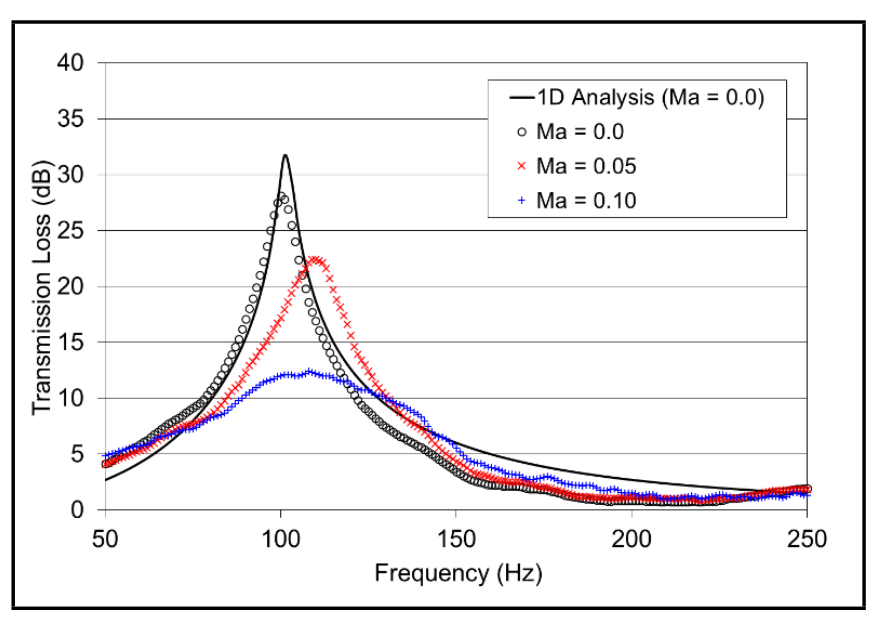

Figure 5. Comparison of TL with flow for no hole open.

frequency with direct flow does not shift to higher frequency. When considering the fact that HRs are mainly intended to reduce the narrow frequency component in intake and exhaust systems of combustion engines, these differences in the peak frequency of the TL can be critical in the design of silencers.

\subsection{HR with Leakage in the Presence of Flow}

Figure 5 shows both the predicted and measured TLs for the HR of Fig. 3 (with no hole open). The HR shown in Fig. 3 is a typical configuration used in automotive exhaust systems. With given geometry and dimensions, in the absence of mean flow, the peak frequency is measured at about $100 \mathrm{~Hz}$ and the magnitude of TL at the peak frequency around $28 \mathrm{~dB}$. The one dimensional prediction of TL without flow in Fig. 5 shows a good agreement with the measured TL. ${ }^{22}$ The effect of flow on the TL of HR given in Fig. 3 is similar to that of the HR with grazing flow shown in Fig. 4. At Ma $=0.05$, compared to the HR with grazing flow, the HR configuration in Fig. 3 shows less decrease in the magnitude at the peak frequency caused by mean flow. With $\mathrm{Ma}=0.1$, the magnitude at the peak frequency significantly decreases by about $16 \mathrm{~dB}$. Thus, the effect of mean flow on TL of HR depends on the flow velocity as well as internal configurations. (a)

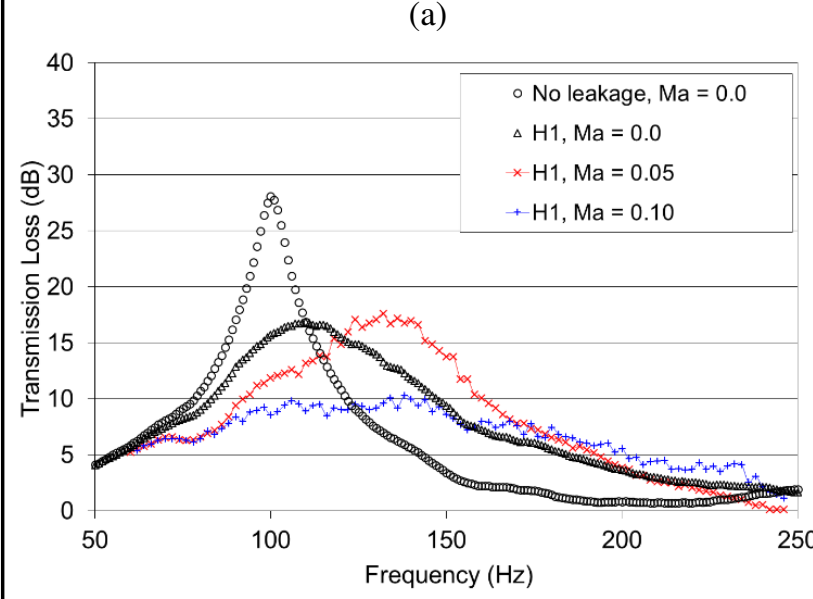

(b)

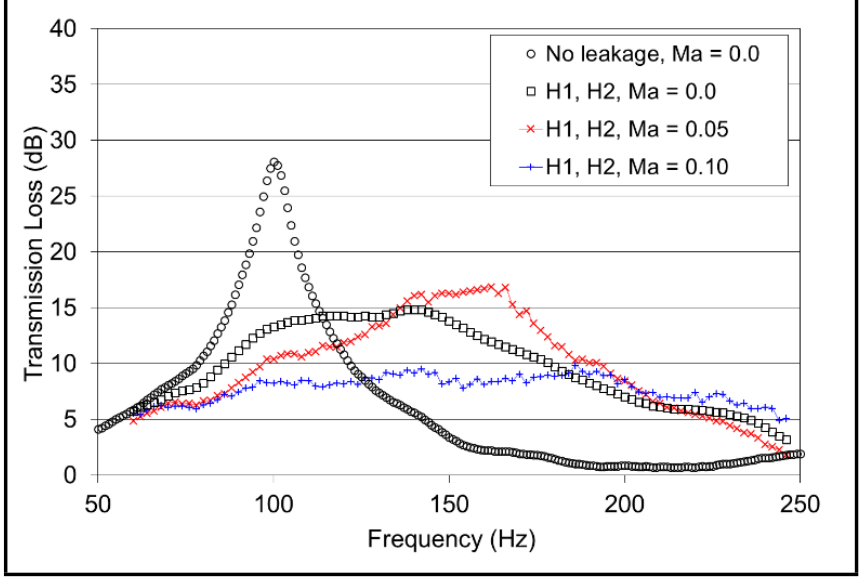

Figure 6. Comparison of TL with flow and: (a) one; (b) two holes open.

Figures 6-8 illustrate the effect of both leakage holes and mean flow on the TL of HR with an internal configuration shown in Fig. 3. Figure 6 (a) shows the measured TL of HR with flow and one hole, H1 in Fig. 3 (b), open. Without flow, the magnitudes of TL already decrease and the peak frequency shift to higher frequency due to the leakage hole, H1, open. Similar trends can be found in Fig. 6 (b) with two holes, H1 and $\mathrm{H} 2$, are open. This phenomenon appears to be similar to HR with grazing flow. However, this phenomenon can be understood with different mechanisms, as demonstrated by Selamet et al. ${ }^{22}$ (effect of leakage) and Kim and Selamet (effect of mean flow). ${ }^{17}$ Figures 6 (a) and (b) show that, for HR with leakage, as flow rate increases to $\mathrm{Ma}=0.05$, the locations of peak TL shift to higher frequency with similar magnitude. However, at $\mathrm{Ma}=0.1$, the magnitude of TL drastically decrease due to the mean flow.

Figure 7 presents measured TL with all 13 holes open with and without flow. In the absence of mean flow, the peak frequency moves from $100 \mathrm{~Hz}$ to $230 \mathrm{~Hz}$ and the bandwidth increases due to the hole opening. At $\mathrm{Ma}=0.05$, unlike other HRs with flow, two peaks at different frequencies appears. Then, at $\mathrm{Ma}=0.1$, only one peak exists, which is similar to the behavior of other HRs with mean flow. At Ma $=0.05, \mathrm{HR}$ behaves as a two degree of freedom system. The magnitude of $\mathrm{TL}$ at resonance frequency is reduced and two peaks appears at both sides of the original resonance frequency. This change 


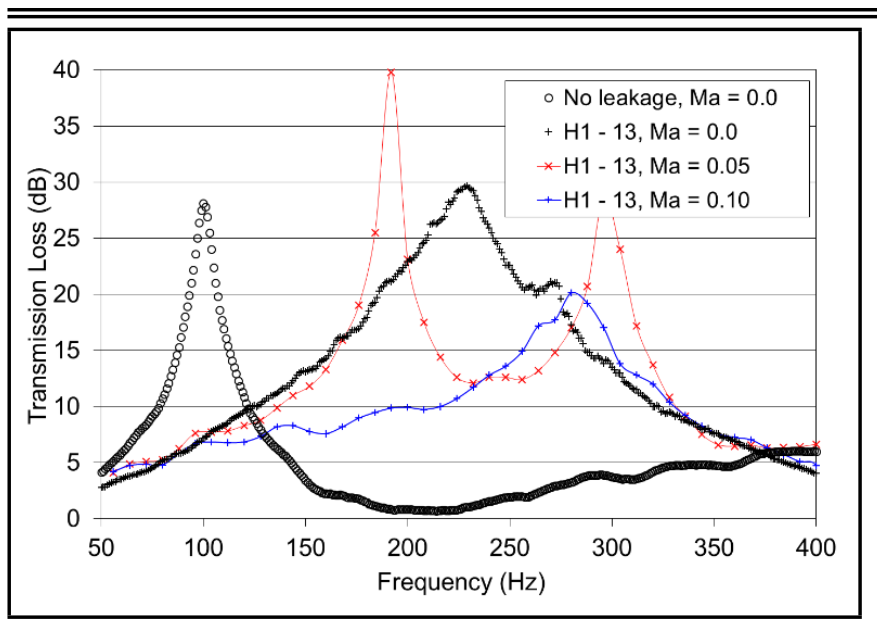

Figure 7. Comparison of TL with flow with 13 holes open.

is due to a certain flow rate that has not been reported by any other previous studies. It is now just assumed that the open holes act as a separate neck at this condition, which is yet to be explained by more extensive numerical and/or experimental studies.

Figure 8 exhibits the effect of holes open in the presence of mean flow as the number of open holes increases. At $\mathrm{Ma}=0.05$ and 0.1 , TLs are illustrated by adding the number of hole by two per each measurement, respectively. Figure 8 (a) shows that at $\mathrm{Ma}=0.05$ the magnitude of the TL substantially decreases and the location of peak frequency increases as discussed earlier. However, as the number of hole openings increases, the first peak TL gradually moves to near $180 \mathrm{~Hz}$ with higher magnitude and at the same time the second peak appears around $300 \mathrm{~Hz}$.

Figure 8 (b) shows that at $\mathrm{Ma}=0.1$, as the number of hole opening increases, the magnitude of the TL appears to be recovered, but at near $280 \mathrm{~Hz}$. The two peak TL phenomena at $\mathrm{Ma}=0.05$ appears to start when 7 holes open. The peak TL magnitude at $\mathrm{Ma}=0.1$ increases at first until the number of hole opening reaches 9 , and then decreases as the number of hole opening exceed 9. Unlike $\mathrm{Ma}=0.05$, the two peak TLs are not observed at $\mathrm{Ma}=0.1$. The effect of flow on the acoustic characteristics of the Helmholtz resonators with drain holes may be crucial for intake and exhaust system design.

\section{CONCLUSION}

The acoustic performance of HRs with different configurations in the presence of mean flow is experimentally investigated using TL. HRs with different flow paths, such as grazing flow and direct flow, are clearly distinguished in TL. HRs with grazing flow shows a decrease in peak TL magnitude and an increase in peak TL frequency. However, HRs with direct flow shows a similar peak TL magnitude at $\mathrm{Ma}=0.05$ and then a decrease at $\mathrm{Ma}=0.1$. In addition, unlike the HR with grazing flow, the peak TL frequency shifts to lower.

HRs with leakage in the presence of mean flow show dramatic changes in peak TL. For HRs with one or two opening holes, as flow rate increases to $\mathrm{Ma}=0.05$, the locations of peak TL shift to a higher frequency with similar magnitude. How- (a)

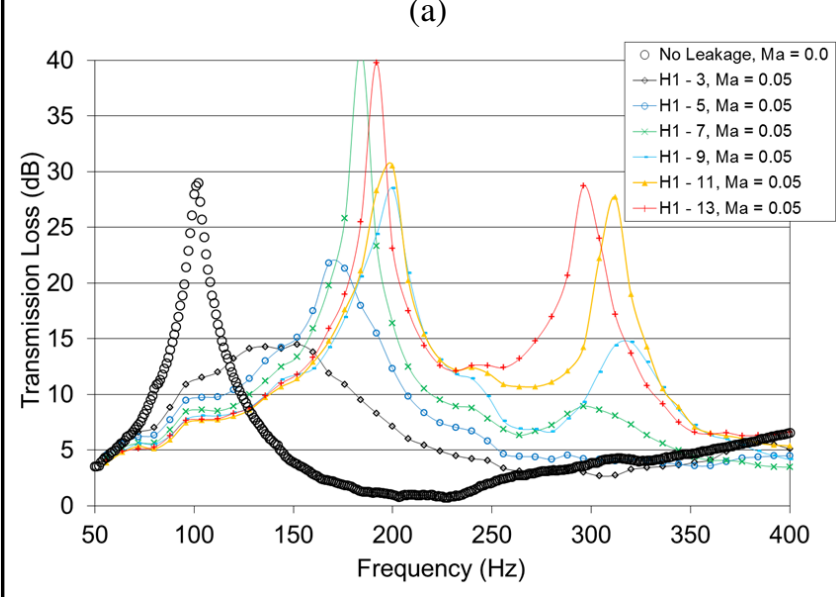

(b)

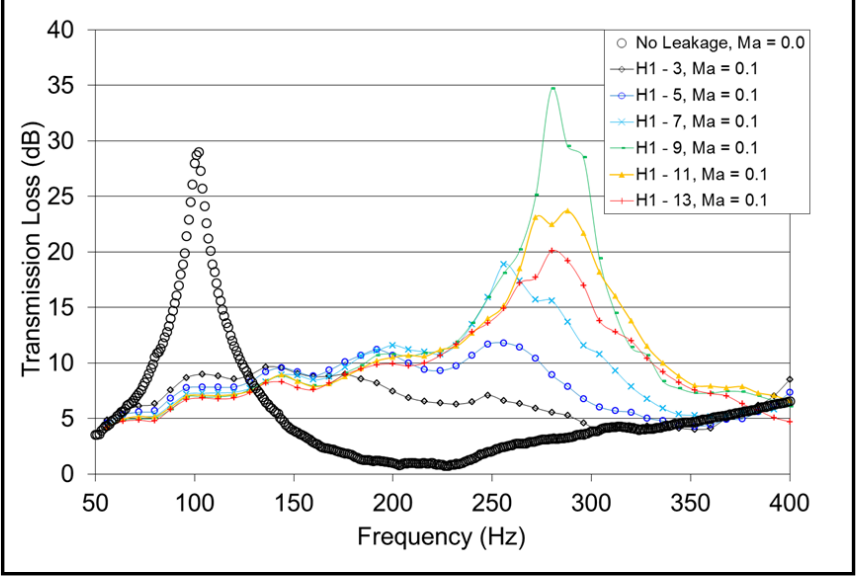

Figure 8. Effect of holes at fixed flow rate: (a) $\mathrm{Ma}=0.05$; (b) $\mathrm{Ma}=0.1$.

ever, at Ma $=0.1$, the magnitude of the TL drastically decreases due to the mean flow. For HRs with more than 7 holes open, it is noteworthy that there is only one peak TL at $\mathrm{Ma}=0.0$ and 0.1 , but two peaks appear at $\mathrm{Ma}=0.05$. This study clearly demonstrates that the effect of mean flow on the acoustic performance of HR strongly depends on the configurations of HR and the conditions of mean flow. Thus, the variation of acoustic performance due to mean flow should be considered in the design of Helmholtz resonators. Future works may involve theoretical and computational approaches corresponding to this work. For instance, the computational approach used by Selamet et al. ${ }^{20}$ can be utilized for the further analysis of this work.

\section{ACKNOWLEDGEMENT}

This profound work is performed by Flow, Engine, and Acoustics Research Laboratories at the Center for Automotive Research in the Ohio State University. Majority of the results are included in the first author's Ph.D. dissertation. ${ }^{26}$

\section{REFERENCES}

1 Panton, R.L. and Miller, J. M. Resonant frequencies of cylindrical Helmholtz resonators, J. Acoust. Soc. Am. 57, 1533-1535 (1975). https://dx.doi.org/10.1121/1.380596 
2 Selamet, A. and Radavich, P. M. Circular concentric Helmholtz resonators, J. Acoust. Soc. Am. 101, 41-51 (1997). https://dx.doi.org/10.1121/1.417986

3 Selamet, A., Ji, Z., and Kach, R.A. Wave reflections from duct terminations, J. Acoust. Soc. Am. 109, 1304 (2001). https://dx.doi.org/10.1121/1.1348298

4 Selamet, A. and Lee, I.J. Helmholtz resonator with extended neck, J. Acoust. Soc. Am. 113, 1975-1985 (2003). https://dx.doi.org/10.1121/1.1558379

5 Selamet, A., Xu, M.B., Lee, I.J., and Huff, N.T. Helmholtz resonator lined with absorbing material, J. Acoust. Soc. Am. 117, 725-733 (2005). https://dx.doi.org/10.1121/1.1841571

6 Tang, S.K. On Helmholtz resonators with tapered necks, J. Sound Vib. 279, 1085-1096 (2005). https://dx.doi.org/10.1016/j.jsv.2003.11.032

7 Xu, M.B., Selamet, A., and Kim, H. Dual Helmholtz resonator, Appl. Acoust. 71, 822-829 (2010). https://dx.doi.org/10.1016/j.apacoust.2010.04.007

8 Kinsler, L.E., Frey, A.R., Coppens, A.B., and Sanders, J.V. Fundamentals of acoustics, John Wiley and Sons, Inc., (1999).

9 Sivian, L.J. Acoustic impedance of small orifices, J. Acoust. Soc. Am. 7, 94-101 (1935). https://dx.doi.org/10.1121/1.1915795

10 Lambert, R.F. Side branch insertion loss in a moving medium, J. Acoust. Soc. Am. 28, 1059-1063 (1956). https://dx.doi.org/10.1121/1.1908555

11 Lambert, R.F. Acoustic fintering in a moving medium, $J$. Acoust. Soc. Am. 28, 1054-1058 (1956).

12 Meyer, E., Mechel, F., and Kurtze, G. Experiments on the influence of flow on sound attenuation in absorbing ducts, J. Acoust. Soc. Am. 30, 165-174 (1958). https://dx.doi.org/10.1121/1.1909527

13 Philips, B. Effects of high-wave amplitude and mean flow on a Helmholtz resonator, NASA report TM X-1582 (1968). https://dx.doi.org/19680013954

14 Baumeister, K.J. and Rice, E.J. Visual study of the effect of grazing flow on the oscillatory flow in a resonator orifice, NASA report TM X-3288 (1975).

15 Hersch, A. and Walker, B.E. Effect of grazing flow on the acoustic impedance of Helmholtz resonators consisting of single and clustered orifices, NASA Contractor Report 3177 (1979).
16 Walker, B.E. and Charwat, A.F. Correlation of the effects of grazing flow on the impedance of Helmholtz resonators, J. Acoust. Soc. Am. 72, 550-555 (1982). https://dx.doi.org/10.1121/1.388035

17 Kim, H. and Selamet, A. Acoustic performance of a Helmholtz resonator with flow, Int. J. Veh. Noise Vib. 7, 285-305 (2011). https://dx.doi.org/10.1504/IJVNV.2011.043191

18 Sun, X., Jing, X., Zhang, H., and Shi, Y. Effect of grazing-bias flow interaction on acoustic impedance of perforated plates, J. Sound Vib. 254, 557-573 (2002). https://dx.doi.org/10.1006/jsvi.2001.4110

19 Selamet, E., Selamet, A., Iqbal, A., and Kim, H. Acoustics of a Helmholtz resonator aligned parallel with flow: A computational study vs. experiments, Int. J. Mater. Mech. Manuf. 1, 210-213 (2013). https://dx.doi.org/ 10.7763/IJMMM.2013.V1.45

20 Selamet, E., Selamet, A., Iqbal, A., and Kim, H. Effect of flow on Helmholtz resonator acoustics: A three-dimensional computational study vs. experiments, SAE Technical paper 2011-01-1521 (2011). https://dx.doi.org/10.4271/2011-01-1521

21 Kim, H., Kang, S.-K., and Lim, Y.-S. Muffler design using transmission loss prediction considering heat and flow, Trans. Korean Soc. Noise Vib. Eng. 24, 600-605 (2014).

22 Selamet, A., Kim, H., and Huff, N.T. Leakage effect in Helmholtz resonators, J. Acoust. Soc. Am. 126, 1142-1150 (2009). https://dx.doi.org/10.1121/1.3183416

23 Chung, J.Y. and Blaser, D.A. Transfer function method of measuring in-duct acoustic properties. I. Theory, J. Acoust. Soc. Am. 68, 907-913 (1980). https://dx.doi.org/10.1121/1.384778

24 Chung, J.Y. and Blaser, D.A. Transfer function method of measuring in-duct acoustic properties. II. Experiment, J. Acoust. Soc. Am. 68, 914-921 (1980). https://dx.doi.org/10.1121/1.384779

25 Seybert, A.F. and Ross, D.F. Experimental determination of acoustic properties using a two-microphone randomexcitation technique, J. Acoust. Soc. Am. 61, 1362-1370 (1977). https://dx.doi.org/10.1121/1.381403

$26 \mathrm{Kim}, \mathrm{H}$. Transmission loss of silencers with flow from a flow-impedance tube using burst signals, Ph.D. Dissertation, The Ohio State University (2011). 